\title{
In Silico Analysis of Baicalin's Binding on Amyloid- $\beta$, Presenilin-1 and Tau Proteins: Validation in a Mouse Model of Cerebral Amyloidosis
}

\author{
Brice Ayissi Owona $^{1 *}$, Arnaud Fondjo Kouam ${ }^{2}$, Zhi yuan Zhang ${ }^{3}$, Hermann J Schluesener ${ }^{3}$ and \\ Paul F Moundipa ${ }^{1}$ \\ ${ }^{1}$ Laboratory of Pharmacology and Toxicology, University of Yaoundé I, Cameroon
}

${ }^{2}$ Medical research and applied Biochemistry Laboratory, Department of Biomedical sciences, University of Buea, Cameroon

${ }^{3}$ Laboratory of Neuropathology of the Nervous Systems, Faculty of Medicine, University of Tuebingen, Germany

*Corresponding author: Vincent Brice Owona Ayissi, Faculty of Science, University of Yaounde 1, PO Box. 812, AEFAS, Cameroon.

To Cite This Article: Brice Ayissi Owona, In Silico Analysis of Baicalin's Binding on Amyloid- $\beta$, Presenilin-1 and Tau Proteins: Validation in a Mouse Model of Cerebral Amyloidosis. 2020 - 10(1). AJBSR.MS.ID.001479. DOI: 10.34297/AJBSR.2020.10.001479.

Received: 非 July 06, 2020; Published: 門 August 26, 2020

\begin{abstract}
The production of amyloid- $\beta$ following activation of presenilin- 1 and accumulation of the hyperphosphorylated tau protein contribute to Alzheimer's disease development. In order to understand Baicalin's effect on AD treatment, we have used in silico studies to predict its binding on amyloid- $\beta$, presenilin- 1 and tau protein receptors. Computational molecular docking was performed using Molegro Virtual Docker software. The scores obtained were $-112.35,-68$ and $-53 \mathrm{Kcal} / \mathrm{mol}$ respectively for the three receptors, indicating potent inhibitory effect of Baicalin on amyloid- $\beta$ (highest binding score). Moreover, Baicalin showed high affinity towards 3jq 5 (amyloid- $\beta$ protein receptor) as it showed thirteen hydrogen bonds with nine amino acids. Contrary to what was observed with amyloid- $\beta$, a lower binding affinity was observed for tau-protein. In addition, to confirm molecular docking results, in vivo studies using APP/PS1 mice (a model of cerebral amyloidosis) treatment with Baicalin showed that it restores behavioural impairment, inhibits the production of amyloid- $\beta$ in the cortex, alongside with the inhibition of macrophage infiltration in the brains of these mice. These findings open new ways for deeper understanding of the amyloid hypothesis in AD and consideration of Baicalin as a promising drug for $\mathrm{AD}$ treatment.
\end{abstract}

Keywords: Alzheimer's disease, Baicalin, In Silico Design, Inflammation, Amyloid- $\beta$

\section{Introduction}

The development of new drugs in the last decade consisted in the association of diseases with the functioning of key proteins which need to be blocked in order to find an effective treatment. This is the case of Alzheimer's Disease (AD), a chronic disorder associated with cognitive decline, loss of memory and death [1]. Genetically, the disease is associated with four genes including Amyloid Precursor Protein (APP), Presenilin-1 (PS1), Presenilin-2 (PS2) and Apolipoprotein E (ApoE). Moreover, mutations associated with APP protein are responsible for the increase of amyloid- $\beta$ peptide deposition in patient's brains. Apart from amyloid- $\beta$, Neu rofibrillary Tangles (NFTs) are also associated with AD. Whether tau proteins contribute to disease worsening is also not fully described. Several drugs have been used in the search of treatment for $\mathrm{AD}$, with unfortunately none that fully treat the disease. Therefore, there is an urgent need of drug discovery to find potential treatments from compounds that are safe and most importantly can fully block the key enzymes involved in $\mathrm{AD}$ development.

Fortunately, Chinese medicine has identified several active compounds which constitute potential lead compounds for AD treatment. Amongst them, Baicalin is a flavonoid compound extracted 
from the plant Scutellaria baicalensis. Recent studies showed that Baicalin inhibits oxidative stress injury in neuronal cells [2]. Baicalin has been established to have potent anti-inflammatory and neuroprotective effects by protecting neurons from microglia-mediated neuroinflammation via suppressing NLRP3 inflammasome and TLR4/NF-KB pathway [3]. The drug was also reported to inhibit $A \beta$-induced microglial cell activation by regulating the JAK2/ STAT3 pathway i006E AD transgenic mice [4]. As far as Amyloid- $\beta$ inhibition is concerned, Baicalin was shown to protect SH-SY5Y cells from oxidative injuries induced by $A \beta 1-42$ aggregation [5]. AD patient's brains revealed the close presence of microglia around $A \beta$ plaques, indicating that neuroinflammation is involved in disease progression. With its anti-inflammatory effects [6], Baicalin appears to be a drug worth investigation due to its multiple effects on disease symptoms.

Structure based-drug design is recognized as a promising tool to discover drug inhibitors and has become a famous way of drug discovery. With this new tool, the drug design process has been facilitated and speeded up considerably. Taking advantage of molecular docking tools, we have first investigated the binding potential of Baicalin on the key enzymes involved in AD initiation and progression. Afterwards, identified target receptors have been validated in vivo using a transgenic mice model of the disease (APP/PS1) which is used to mimic human manifestations of AD.

\section{Materials and Methods}

\section{Molecular Docking}

Ligand preparation: Baicalin was identified from PubChem chemical database. The structure was drawn using Molview software (Figure 1) and energy minimization performed using the MM2 force field and saved as a. MOL format. The missing charges and hybridization states of Baicalin structure were assigned with the help of the MVD software.

Protein preparation: The 3D structure of different receptors was retrieved from the Protein Data Bank (PDB) (http://www. rcsb.org). We have chosen respectively: Amyloid- $\beta$ (PDB ID: 1BA4), presenilin-1 (PDB ID: 5A63) and tau protein (PDB ID:6NK4). The proteins had one or two polypeptides and were co-crystallized with ligands. The targets were visually inspected, and reference ligands identified for each receptor. Receptors were prepared for docking by removal of water molecules, ligands, cofactors and assigning bonds, bond order, hybridization and charges using the MVD software [7].

Docking search algorithm and scoring functions: MVD uses PLP (Piecewise Linear Potential) algorithm as scoring function for computational screening. In this study, the MolDock simplex evolution search algorithm was used for docking. In practice, the number of receptor cavities was limited to four and using the cavity prediction wizard, the cavity with large volume was selected. Docking of Baicalin in different receptors was performed and the best poses generated were used based on the docking scores (expressed in $\mathrm{Kcal} / \mathrm{mol}$ ).

Parameters for scoring functions: MVD software uses two scoring functions: the MolDock score and the ReRank score, where MolDock score is an E score (docking scoring function) defined as: $\mathrm{E}$ score $=\mathrm{E}$ inter $+\mathrm{E}$ intra.

A. E inter: Sum of ligand-protein interaction energy, ligand-water interaction energy and ligand-cofactor interaction energy [8].

B. E intra: Internal energy of the ligand.

ReRank score provides an estimation of the ligand-receptor interaction strength [9].

\section{Animals}

For in vivo experimentation, we have used male APP/PS1 mice which were a donation from Prof Jucker, Hertie-Institute, Tuebingen, Germany. Males APP/PS1-21 mice were bred with wild-type C57BL/6J (Charles River Germany). Thereafter, we have used PCR reaction for genotyping of offspring with the help of specific primers for the APP-sequence (Forward:" GAATTCCGACATGACTCAGG", Reverse: "GTTCTGCTGCATCTTGGACA"). The experiments were conducted after approval by the local institutional animal care committee and according to the German Animal Welfare Act (TierSchG) of 2006.

\section{Materials}

Baicalin was purchased from Carbosynth Ltd. (Compton, Berkshire, UK). Baicalin was suspended in 1\% Carboxymethylcellulose (CMC, Blanose, Hercules-Aqualon, Düsseldorf, Germany) and administered by gavage to animals at a dose of $20 \mathrm{mg} / \mathrm{kg}$. Control mice received an equivalent volume of CMC.

\section{Treatment with Baicalin}

APP/PS1 transgenic mice were divided into two groups ( $n=6$, three females and three males at the age of 5 months). Mice were treated for 21 days as follows. A) Baicalin daily oral administration, $20 \mathrm{mg} / \mathrm{kg}$ bw, B) Vehicle-treated used as control in this study.

\section{Nest construction assay}

We used the next construction assay with few modifications to evaluate the deficits in social behaviour of APP/PS1 mice and the potential effect of Baicalin following treatment. Mice were individually housed for 24 hours in clean plastic cages with $1 \mathrm{~cm}$ of wood chip bedding. Individual cages were then supplied with paper towel torn into $5 \times 5 \mathrm{~cm}$ squared pieces, two hours prior to the onset of the dark phase. On the following day, different cages were inspected for nest construction. Test scores were examined by three independent individuals (who were not aware of the treatment procedure) by observing the paper Powel with the help of a three-point scale system: 
A. No biting or tears on the paper,

B. Moderate biting and/or tears on the paper but no coherent nest

C. The vast majority of paper torn into pieces and grouped into a corner of the cage.

\section{Immunohistochemistry and image analysis}

Mice treated with Baicalin and control mice were sacrificed after 21-days of treatment. Thereafter, they were perfused intracardially with 4\% PBS (paraformaldehyde). Mice brains were quickly removed and post-fixed in $4 \%$ PBS overnight at $4^{\circ} \mathrm{C}$. Thereafter, brains were cut into two hemispheres, embedded in paraffin, serially sectioned $(3 \mu \mathrm{m})$ and mounted on silan-covered slides. Hemispheres sections were stained by immunohistochemistry, using the following antibodies: $\beta$-amyloid (1:100; Abcam, Cambridge, UK) for $A \beta$ deposition, Iba-1 (1:200; Wako, Neuss, Germany) for activated microglia.

In the next experiment, hemisphere sections were examined and photographed using a light microscope (Nikon Coolscope, Nikon, Düsseldorf, Germany). All sections were randomly numbered and analysed by two independent observers, who were not aware of different treatments. At the end, amyloid- $\beta$ plaques, APP plaque-like staining, and Iba- $1^{+}$cells in the cortex and hippocampus were counted. During observation, APP plaque staining was localized on cells or outside cells with a plaque-like expression pattern. Moreover, the number of APP plaques was also counted according to the standard of $A \beta$. Images of hemisphere cross-sections were captured with fixed parameters, the cortex and hippocampus on the pictures were outlined and analysed using the software MetaMorph Offline 7.1, Toronto, Canada.

\section{Statistical analysis}

Differences of plaque/cell counts and nest construction data were analysed using the unpaired t-test (Graph Pad Prism 6.0 software). For all statistical analysis, significance levels were set at $\mathrm{P}<0.05$.

\section{Results}

\section{Molecular Docking of Baicalin with key Receptors involved in Alzheimer's Disease}

After performing docking analysis, the results obtained in Table $1 \& 2$ and Figure 2 indicate that Baicalin fits perfectly at the active sites of 3jq5, 5a63 and $6 \mathrm{nk} 4$, respectively. The bindings involved for 3jq5 (amyloid- $\beta$ ) complex interactions with seven amino acids including Leu 2, Lys 6, Phe 5, Phe 65, Trp 19, Tyr 3 and Tyr 64. Moreover, the binding of Baicalin is stabilized by 13 hydrogen bonds between baicalin and the receptor. For Presenilin-1, amino acids involved in the binding process included Arg 657, Asp 655, Glu 68, Lys 654, Phe 218, Ser 67, Lys 101, Ser 102, Ser 104 and Val 103 , and the binding was stabilized with 6 hydrogen bonds. As far as tau protein is concerned, amino acids included Asn 6, Gln 3, Ile 4, Ile 5, Lys 1, and Val 2 with 3 hydrogen bonds between the drug and the receptor's binding pocket.

Table 1: The Docking scores of Baicalin with 3jq5, $5 a 63$ and 6nk4 receptor proteins.

\begin{tabular}{|c|c|c|c|c|c|}
\hline S. No & Ligand & Protein & MolDock (Kcal/mol) & ReRank(Kcal/mol) & Hbond(Kcal/mol) \\
\hline 1 & Baicalin & 3jq5- & -112.35 & -133.35 & -5.435 \\
\hline 2 & Baicalin & 5a63-Presenilin & -68.5368 & -57.1536 & -5.00 \\
\hline 3 & Baicalin & 6nk4-tau & -53.455 & -58.051 & -4.640 \\
\hline
\end{tabular}

Table 2: The total interaction energy of Baicalin docked with amyloid- $\beta$, presenilin1 and tau protein receptors.

\begin{tabular}{|c|c|c|c|c|}
\hline S. No & Molecule & Residue & ID & Total Energy of Interaction (in Kcal/mol) \\
\hline 1 & 3jq5 & Leu & 2 & -17.672 \\
\hline 2 & 3jq5 & Lys & 6 & 8.72528 \\
\hline 3 & 3jq5 & Phe & 5 & -0.33162 \\
\hline 4 & 3jq5 & Phe & 19 & -0.97323 \\
\hline 5 & 3jq5 & Trp & 3 & -17.9038 \\
\hline 6 & 3jq5 & Tyr & 64 & -20.8586 \\
\hline 7 & $3 j q 5$ & Tyr & 657 & -0.44572 \\
\hline 1 & $5 \mathrm{a} 63$ & Arg & 655 & -7.29971 \\
\hline 2 & $5 \mathrm{a} 63$ & Asp & 37 & -0.58562 \\
\hline 3 & $5 \mathrm{a} 63$ & Glu & 68 & -4.85773 \\
\hline 4 & $5 \mathrm{a} 63$ & Gly & 654 & -0.80031 \\
\hline 5 & $5 \mathrm{a} 63$ & Lys & 218 & -4.68293 \\
\hline 6 & $5 \mathrm{a} 63$ & Phe & 67 & -9.08734 \\
\hline 7 & $5 \mathrm{a} 63$ & Ser & & -4.67108 \\
\hline
\end{tabular}




\begin{tabular}{|c|c|c|c|c|}
\hline 8 & $5 \mathrm{a} 63$ & Lys & 101 & -8.63399 \\
\hline 9 & $5 \mathrm{a} 63$ & Ser & 102 & -3.36299 \\
\hline 10 & $5 \mathrm{a} 63$ & Ser & 104 & -3.75729 \\
\hline 11 & $5 \mathrm{a} 63$ & Val & 103 & -8.48139 \\
\hline 1 & $6 \mathrm{k} 4$ & Asn & 3 & -5.52614 \\
\hline 2 & $6 \mathrm{nk4}$ & Gln & 4 & -22.5 \\
\hline 3 & $6 \mathrm{nk} 4$ & Ile & 5 & -21.5953 \\
\hline 4 & $6 \mathrm{nk} 4$ & Ile & 1 & -32.077 \\
\hline 5 & $6 \mathrm{nk4}$ & Lys & 2 & -12.4841 \\
\hline 6 & $6 \mathrm{nk} 4$ & Val & -2.92494 \\
\hline
\end{tabular}

A

B

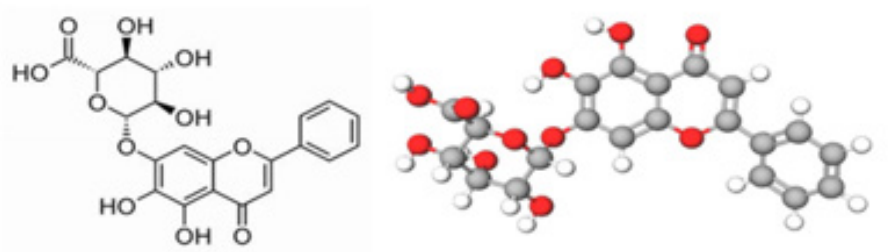

Figure 1: (A) Chemical (B) 3D structures of Baicalin.

A

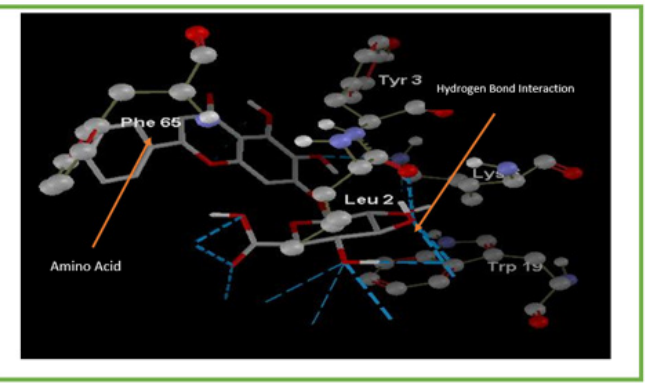

B

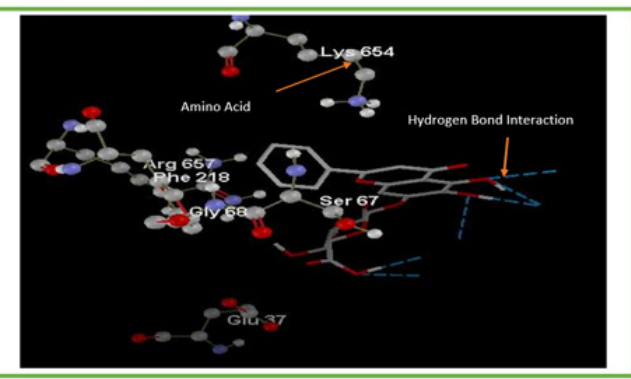

C

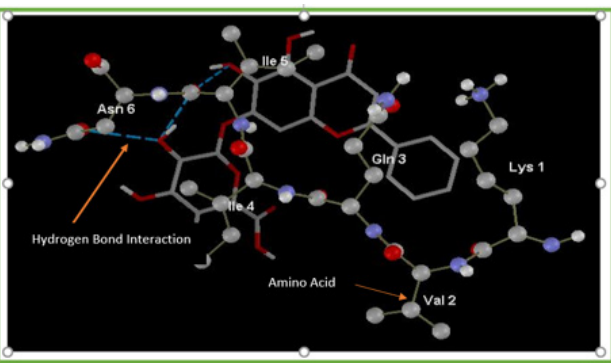

Figure 2: Interaction of Baicalin with active cavities of (A) Amyloid- $\beta$, (B) Presenilin1 and (C) Tau proteins. 
The docking scores in Kcal/mol indicated that the highest binding for Baicalin is obtained with amyloid- $\beta$ (MolDock score: -112.35, ReRank score: -133.35), followed by Presenilin-1 (MolDock score: -68.5368, ReRank score: -57.1536), and a lower binding was obtained for tau protein (MolDock score: -53.4550). Baicalin showed potent inhibitory effect greater with amyloid- $\beta$ than with tau-protein.

\section{Effect of Baicalin Treatment on Nest Construction Capacity}

Transgenic APP/PS1 mice were tested to evaluate the effect of Baicalin treatment on behavioural impairment. Following treatment, we observed an improvement of the nesting capacities of treated mice. As shown in Figure 3, no significant difference was observed between treated and non-treated mice at day 1 (control= 1.6., Baicalin $=2.00, n=6$ ). Interestingly, after 21 days of treatment, a significant difference between treated and non-treated mice was observed (control=1.7, Baicalin=2.8, n=6) with an immediate chew- ing and tearing of the paper towel fragments. Contrary to non-treated mice, APP/PS1 transgenic mice chewed the paper towels which were found grouped at one corner of the cage (Figure 3C).

\section{Effect of Baicalin on Amyloidosis and Neuroinflammation}

Mice receiving no treatment did not show any significant difference in amyloid- $\beta$ deposition and Iba- 1 expression. Baicalin oral treatment improved neuropathological changes in comparison to non-treated mice. It appears from Figure 4 that Baicalin significantly reduced the inflammation in mice brains by limiting activation of microglia cells both in the cortex and hippocampus (control= $198 \pm 0.341$, Baicalin $=140 \pm 0.564, \mathrm{p}<0.05, \mathrm{n}=6$ and hippocampus control $=59 \pm 0.811$, Baicalin=36 $\pm 0.465, p<0.05, n=6$ ). On the other hand, Baicalin also significantly reduced plaque number only in the cortex (control $=78 \pm 0.84$, Baicalin $=37 \pm 0.310, \mathrm{p}<0.05, \mathrm{n}=6$; and hippocampus control $=4 \pm 0.015$, Baicalin $=4 \pm 0.028, \mathrm{p}<0.05, \mathrm{n}=6$ ) (Figure 5).

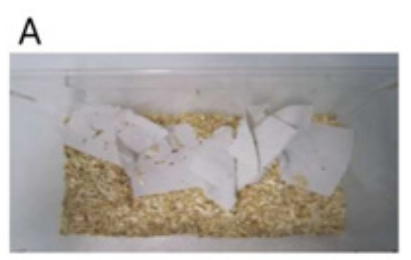

\section{C}

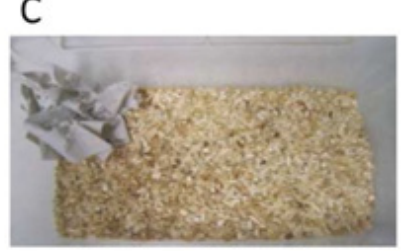

B

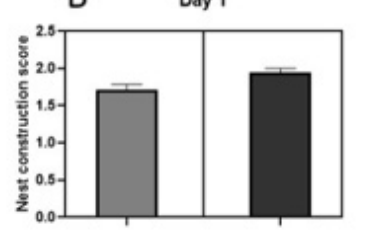

Eacalin

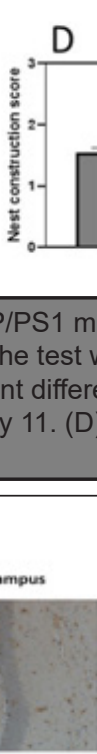

Day 21

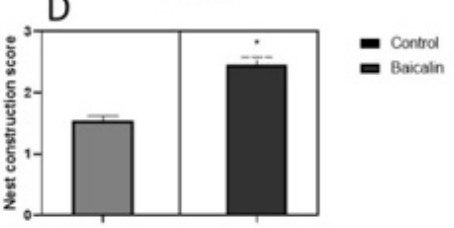

Figure 3: Effect of Baicalin treatment on nest construction capacity. APP/PS1 mice were treated orally with Baicalin for 21 days. Transgenictreated and naïve mice were later run assessed for nesting behaviour. The test was scored using a three-point scaling system (see materials and methods). (A): Observation of the nest building at day 1. (B) No significant difference between Baicalin and the control group was observed right at the beginning of treatment. $(C)$ : Observation of the nest building at day 11. (D): At day 11, a significant difference between the Baicalin and control group was observed after 11 days of treatment. $P<0.05$
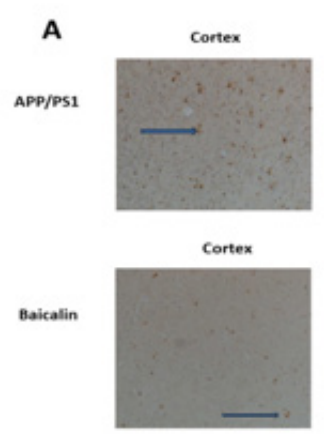

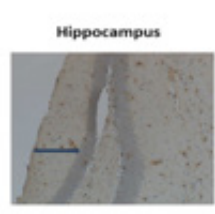

Hippocampus

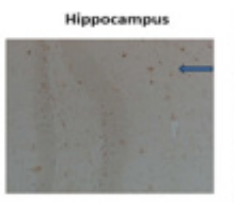

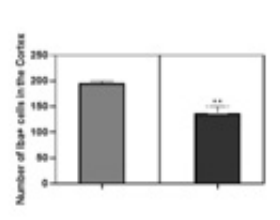

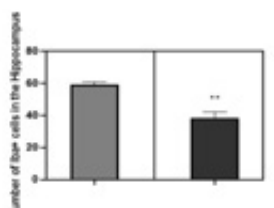

B

ansos

$=$

Figure 4: Effect of Baicalin on microglial activation. APP/PS1 mice were either treated with Baicalin or the vehicle (carboxymethylcellulose). After 21 days of treatment by gavage, brains were dissected and analysed by immunohistochemistry. Iba+ cells were counted and represented in a graph. The unpaired t-test was used to calculate the differences of cell counts between treatment group and control, with significance set at $p<$ 0.05 . 


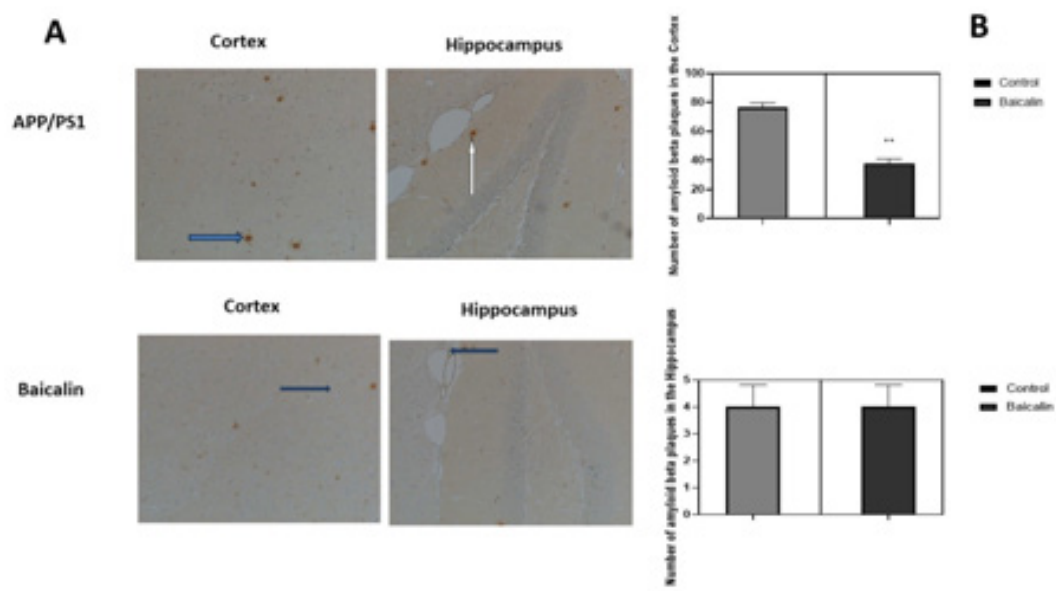

Figure 5: Effect of Baicalin on amyloid- $\beta$ deposition in mice cortex and hippocampus. APP/PS1 mice were either treated with Baicalin or the vehicle (carboxymethylcellulose). After 21 days of treatment by gavage, brains were dissected and analysed by immunohistochemistry. Plaques of amyloid- $\beta$ were counted and represented in a graph. The unpaired t-test was used to calculate the differences of plaque counts between treatment group and control, with significance set at $p<0.05$. A reduction of amyloid plaques was observed in the cortex of transgenic mice. No significant effect was observed in the hippocampus.

\section{Discussion}

Baicalin is a bioactive flavone with reported anti-inflammatory and neuroprotective effects [10]. Consumption of foods rich in flavonoids can affect cognitive function. Indeed, several flavonoids have been reported to reverse deficits in cognition and inhibit the development of $\mathrm{AD}$ [11]. We have conducted this research in order to find out the binding capacity of Baicalin on amyloid- $\beta$, presenilin-1 and tau protein. The mentioned proteins are reported to play a critical role in $\mathrm{AD}$ development and pathogenesis. Before conducting in vivo experimentations, it is important to analyse the binding effects of drugs with their receptor proteins in silico in order to have an idea on how well the drug will bind to its target in vivo. Moreover, the development and deposition of amyloid- $\beta$ plaques and the formation of neurofibrillary tangles of tau protein are reported to play a major role in the disease. Our aim was to evaluate the binding of Baicalin on these three proteins with major role in AD.

In our study, Baicalin was found bound to the active cavities of different receptor proteins and showed binding with amino acids of the binding pocket. The binding energy of Baicalin with amino acids (Leu2, Lys6, Phe5, Phe65, Trp19, Tyr3 and Tyr64) were compared with the binding energy of Presenilin-1 with amino acids (Arg657, Asp655, Glu68, Lys654, Phe218, Ser67, Lys101, Ser102, Ser104 and Val103) and of tau protein with amino acids (Asn6, Gln3, Ile4, Ile5, Lys1 and Val2) (Table 2). The results showed that Baicalin binds to amyloid- $\beta$ with the highest score and strongest energy (MolDock score: $-112.35 \mathrm{Kcal} / \mathrm{mol}$; ReRank score: $-133.35 \mathrm{Kcal} / \mathrm{mol}$ ), followed by Presenilin-1(MolDock score: -68.5368; ReRank score: -57.1536) and tau protein (MolDock score: $-53.4550 \mathrm{Kcal} / \mathrm{mol}$ ). Analysis of binding interactions revealed that Baicalin showed high affinity for amyloid- $\beta$ as Baicalin showed thirteen hydrogen bonds with seven amino acids, in comparison to 6 and 3 hydrogen bonds with Presenilin- 1 and tau protein respectively. These results confirm the amyloid- $\beta$ theory on $\mathrm{AD}$ stating that amyloid- $\beta$ is mostly responsible for disease development and therefore, research focusing at finding inhibitors of amyloid- $\beta$ formation and deposition in the brain in vivo could be an effective strategy to cure the disease.

In order to confirm the results obtained following molecular docking, we tried to study the effect of Baicalin on amyloid- $\beta$ in vivo. We have used transgenic mice APP/PS1, a mouse model of AD amyloidosis exhibiting remarquable elevation of amyloid- $\beta$ associated with behavioural deficiencies. Moreover, these mice express the Swedish mutation of APP and the L166P mutation of human presenilin-1. In addition, these mice show plaque formation at 2 months in the neo-cortex and 4 months in the hippocampus [12]. Impairment in nesting construction is another hallmark of AD and this is used to mimic behavioural deficits in vivo. In this study, Baicalin was administered to mice in vivo for a period of 21 days. The results obtained showed that after this treatment time, mice treated with Baicalin showed an increase in nesting score in comparison to control mice, indicating the potential of the drug to improve behavioral impairments in these mice. The same effect obtained with Baicalin was noted with other flavonoid and plant compounds such as Zerumbone, Herperidin and Icariin [13-15]. Another hallmark of $\mathrm{AD}$ is the deposition and accumulation of amyloid- $\beta$ in the cortex and hippocampus. The results obtained showed a significant reduction of plaque number in the cortex, but, no significant reduction was observed in the hippocampus of mice. From the results obtained, it appears that the time of treatment with Baicalin may have an effect on its efficiency in plaque reduction. 
In a recent study, it was reported that treatment of APP/PS1 mice with Baicalin for 11 days did not show any significant effect in hippocampus and cortex [16]. Increasing the number of days of treatment may therefore considerably improve the treatment, at least by reducing plaque accumulation in the cortex. Accumulation of amyloid- $\beta$ always results in neuroinflammation with activation of microglia which in turn potentiate inflammation in the brain. Therefore, anti-inflammatory compounds in the brain could be good candidate for AD treatment. Baicalin is a well-known anti-inflammatory drug with in vitro antioxidant and immunomodulatory effects. Treatment of mice with the drug showed a reduction of inflammation in the cortex and hippocampus of treated mice in comparison to the control mice. This was evaluated in this study by measuring the expression of Iba+ cells, a marker of microglia inflammation. In silico inhibition of amyloid- $\beta$ therefore corroborate in vivo observations, suggesting that the binding of Baicalin with high score and high affinity to its receptor is responsible for the observed improvement in nesting scores, amyloid plaque number reduction in the cortex and the resulting reduction inflammation in mice brains.

\section{Conclusion}

This study investigated the capacity of Baicalin to bind amyloid- $\beta$, presenilin- 1 and tau protein, which are proteins involved in the initiation and development of AD. In silico studies showed that Baicalin binds preferably to amyloid- $\beta$ with the highest and strongest energy. In vivo, Baicalin showed inhibitory effect on $A \beta$ accumulation in the cortex and restored behavioral impairments in mice. Therefore, further investigation of Baicalin as a potential drug for $\mathrm{AD}$ are needed in order to open new ways for drug discovery.

\section{Acknowledgement}

The authors would like to thank Prof. M. Jucker for providing male transgenic APP/PS1-(21) mice. The study was supported by the Deutscher Akademischer Austausch-Dienst (DAAD). The authors declare there is no conflict of interest.

\section{References}

1. Feng Y, Wang X (2012) Antioxidant therapies for Alzheimer's disease. Oxidative Medicine and Cellular Longevity 2012: 472932.

2. Wang ZY, Liu JG, Li H, Yang HM (2016) Pharmacological Effects of Active Components of Chinese Herbal Medicine in the Treatment of Alzheimer's Disease: A Review. American Journal of Chinese Medicine 44(8): 15251541.
3. Jin X, Liu MY, Zhang DF, Zhong X, Du K, et al. (2019) Baicalin mitigates cognitive impairment and protects neurons from microglia-mediated neuroinflammation via suppressing NLRP3 inflammasomes and TLR4/ NF- $\kappa B$ signaling pathway. CNS Neuroscience and Therapeutics 25(5): 575-590.

4. Xiong J, Wang C, Chen H, Hu Y, Tian L, et al. (2014) A $\beta$-induced microglial cell activation is inhibited by baicalin through the JAK2/STAT3 signalling pathway. International Journal of Neuroscience 124(8): 609-620.

5. Yin F, Liu J, Ji X, Wang Y, Zidichouski J, Zhang J (2011) Baicalin prevents the production of hydrogen peroxide and oxidative stress induced by $A \beta$ aggregation in SH-SY5Y cells. Neuroscience Letters 492(2): 76-79.

6. Mandrekar Colucci S, Landreth GE (2012) Microglia and Inflammation in Alzheimer's Disease. CNS \& Neurological Disorders-Drug Targets 9(2): 156-167.

7. Dubey K, Dubey R (2020) Computation screening of narcissoside a glycosyloxyflavone for potential novel coronavirus 2019 (COVID-19) inhibitor. Biomedical Journal.

8. Heble NK, Mavillapalli RC, Selvaraj R, Jeyabalan S (2016) Molecular docking studies of phytoconstituents identified in Crocus sativus, Curcuma longa, Cassia occidentalis and Moringa oleifera on thymidylate synthase-An enzyme target for anti-cancer activity. Journal of Applied Pharmaceutical Science 6(12): 131-135.

9. Madhuri M, Prasad C, Rao Avupati V (2014) In Silico Protein-Ligand Docking Studies on Thiazolidinediones as Potential Anticancer Agents. International Journal of Computer Applications 95(6): 13-16.

10. Chen C, Li X, Gao P, Tu Y, Zhao M, et al. (2015) Baicalin attenuates Alzheimer-like pathological changes and memory deficits induced by amyloid $\beta_{1-42}$ protein. Metabolic Brain Disease 30: 537-544.

11. Williams RJ, Spencer JPE (2012) Flavonoids, cognition, and dementia: Actions, mechanisms, and potential therapeutic utility for Alzheimer disease. Free Radical Biology and Medicine 52(1): 35-45.

12. Harach T, Marungruang N, Duthilleul N, Cheatham V, Mc Coy KD, et al. (2017) Reduction of Abeta amyloid pathology in APPPS1 transgenic mice in the absence of gut microbiota. Scientific Reports 7: 1-15.

13. Li C, Zug C, Qu H, Schluesener H, Zhang Z (2015) Hesperidin ameliorates behavioral impairments and neuropathology of transgenic APP/PS1 mice. Behavioural Brain Research 281: 32-42.

14. Li L, Wu XH, Zhao XJ, Xu L, Pan CL, et al. (2020) Zerumbone ameliorates behavioral impairments and neuropathology in transgenic APP/PS1 mice by suppressing MAPK signalling. Journal of Neuroinflammation 17: 61.

15.Zhang ZY, Li C, Zug C, Schluesener HJ (2014) Icariin ameliorates neuropathological changes, TGF- $\beta 1$ accumulation and behavioral deficits in a mouse model of cerebral amyloidosis. PLoS ONE 9(8): 104616.

16. Li C, Meng P, Zhang B, Kang H, Wen H, et al. (2019) Computer-aided identification of protein targets of four polyphenols in Alzheimer's Disease (AD) and validation in a mouse AD model. Journal of Biomedical Research 33(2): 101-112. 